\title{
Effects of Vitiation on the Heat Release Rate in Mechanically- Ventilated Compartment Fires
}

\author{
STEPHANE MELIS and LAURENT AUDOUIN \\ Institut de Radioprotection et de Sûreté Nucléaire (IRSN) \\ Centre de Cadarache, $\mathrm{BP} \mathrm{n}^{\circ} 3,13115$, St Paul-Lez-Durance Cedex- France
}

\begin{abstract}
The prediction of the heat release rate in compartment fires is still a challenging question due to the feedback of the environmental variables on the burning rate. A set of large-scale, mechanically-ventilated pool fire experiments supports the present analysis. Most of these tests yielded a quasi steady-state with limited pyrolysis compared to well-ventilated conditions. From a well-stirred reactor approach, the effect of vitiation on the steady-state pyrolysis rate is discussed. The Global Equivalence Ratio concept, expressed as $\Phi=A_{f} \dot{m}_{\infty}^{\prime \prime} \Delta H_{c} / q_{0} Y_{\infty} \Delta H_{O 2}$, is introduced to classify the experiments. It also appears to be the main factor in determining the under-ventilated pyrolysis rate whenever the airflow inlet is situated high in the fire room. Whenever the inlet position is low, however, the oxygen concentrations tend to be stratified and the well-stirred reactor approach fails to predict the pyrolysis rate. In both cases, a zone modelling approach including the dependency of pyrolysis on the oxygen content near the flame provides a better estimation of the fuel mass loss rate and hence of the heat release rate.
\end{abstract}

KEYWORDS: modelling, compartment fires, heat release rate.

\section{NOMENCLATURE LISTING}

$\begin{array}{ll}A_{f} & \text { pool area }\left(\mathrm{m}^{2}\right) \\ h_{f} & \text { flame height }(\mathrm{m}) \\ \dot{m}^{\prime \prime} & \text { pyrolysis rate }\left(\mathrm{kg} / \mathrm{m}^{2} / \mathrm{s}\right) \\ P & \text { pressure }(\mathrm{Pa}) \\ q & \text { ventilation mass flow-rate }(\mathrm{kg} / \mathrm{s}) \\ R & \text { airflow resistance }\left(\mathrm{m}^{-4}\right) \\ S & \text { oxygen-to-fuel mass ratio } \\ T & \text { room temperature }(\mathrm{K}) \\ V & \text { compartment volume }\left(\mathrm{m}^{3}\right) \\ Y & \text { oxygen mass fraction }(\mathrm{kg} / \mathrm{kg}) \\ \mathrm{Z} & \text { elevation }(\mathrm{m})\end{array}$

(a) in well-ventilated conditions.

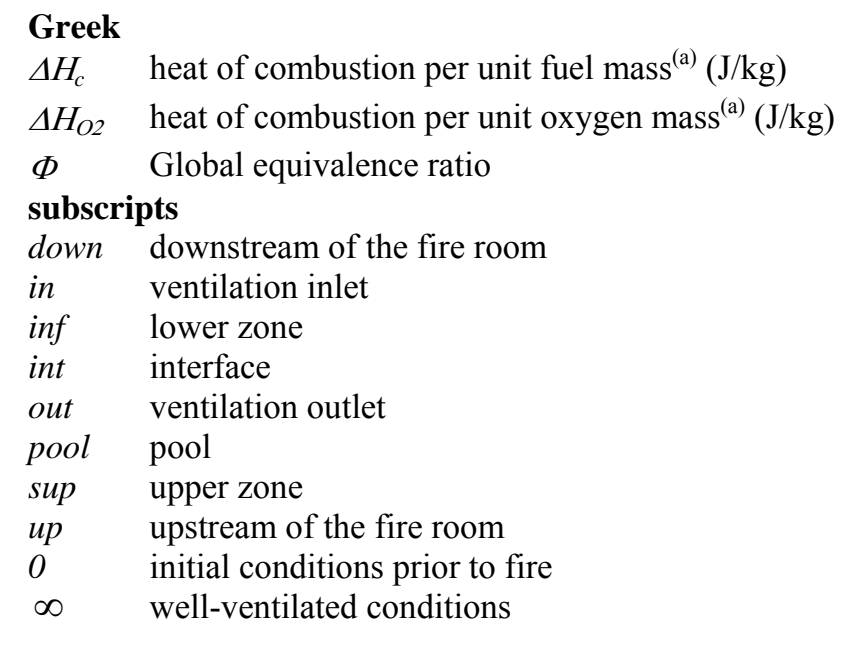

$\begin{array}{ll}\text { Greek } & \\ \Delta H_{c} & \text { heat of combustion per unit fuel mass } \\ \Delta H_{O 2}(\mathrm{a}) & \text { heat of combustion per unit oxygen } \operatorname{mass}^{(\mathrm{a})}(\mathrm{J} / \mathrm{kg}) \\ \Phi & \text { Global equivalence ratio } \\ \text { subscripts } \\ \text { down } & \text { downstream of the fire room } \\ \text { in } & \text { ventilation inlet } \\ \text { inf } & \text { lower zone } \\ \text { int } & \text { interface } \\ \text { out } & \text { ventilation outlet } \\ \text { pool } & \text { pool } \\ \text { sup } & \text { upper zone } \\ \text { up } & \text { upstream of the fire room } \\ 0 & \text { initial conditions prior to fire } \\ \infty & \text { well-ventilated conditions } \\ & \end{array}$

\section{INTRODUCTION}

As previously stated by Babrauskas [1], the heat release rate appears to be the most important variable in fire hazard. Nowadays, the heat release rate of most combustibles can be obtained quite readily from small, medium and large-scale calorimeters under well-ventilated conditions [2]. Besides, introducing the heat release rate in most compartment-fire modelling yields generally acceptable prediction of the major features of a fire scenario, such as the temperature or the release of toxic species. However, the knowledge of the open-atmosphere heat-release rate is not sufficient to predict the time evolution of the actual fire heat release rate in rooms because of the strong interaction existing between combustion and ventilation. The enclosure modifies, often in a significant manner, environmental variables such as the oxygen content, the wall and gas temperatures and generally the heat fluxes to the fuel surface. In turn, these variables can affect both the fuel mass loss rate and the heat of combustion that are the two components of the heat release rate. Modelling this last influence is still a great challenge for fire codes, as Utiskul et al. have recently shown [5]. Moreover, as they influence the mass loss rate, the environmental variables also play a 
role in the fire duration. This latter variable is also an important determinant of fire hazard as safety devices such as firebreak doors are usually designed to resist only during a limited time.

In the nuclear industry, compartments are generally sealed from one another while connected to a ventilation network. This ventilation system provides a suitable pressure cascade to prevent any accidental radioactive leak within the compartment from escaping to the open atmosphere. The rooms where radioactive materials are present are under-pressurized and the outgoing air flows are gathered and filtered thanks to high-efficiency air-cleaning devices. This particular design is of some consequence in case of a fire as the low ventilation level usually leads to under-ventilated fires. In such a ventilation regime, the influence of the environmental variables on the heat release rate has been reported to be most important $[6][10][11]$.

The influence of vitiation on the heat release rate, and particularly on the fuel mass loss rate, has been investigated previously (e.g. [3][5][10][11][12]). However, most of the studies involved small-scale experiments, in which the temperature effect and the oxygen effect can be discriminated only with difficulty. A set of real-scale, mechanically-ventilated pool fire experiments provides the basis of the present study. These experiments were carried out by the French "Institut de Radioprotection et de Surreté Nucléaire", some of them in collaboration with AREVA-NC [13][14] or in the frame of the preparation of the present OECD PRISME project [12]. These tests yielded a quasi steady-state with reduced pyrolysis and a limited increase of gas temperatures. A well-stirred reactor approach is used to investigate the effect of the vitiation on the steady-state mass loss rate. In addition,, the experimental results are compared to a zone model in which Peatross and Beyler correlation [3] was introduced. The implications in terms of mass loss rate and combustion efficiency are discussed.

\section{LITERATURE REVIEW}

From more than 25 years, many investigators were interested in the influence of the confinement on the heat release rate. Using natural ventilation conditions, Takeda et al. [7] investigated the behaviour of liquid-fuel compartment fires in cubic rooms (sides from 0.15 to $0.7 \mathrm{~m}$ ) with a single rectangular opening, in which one liquid fuel tray of methanol $\left(0.15 \times 0.15 \mathrm{~m}^{2}\right)$ was centrally located. For this pool fire, they observed both unstable and stable oscillatory combustion (so-called "critical phenomenon"). This regime was dependent on the compartment size and on the ventilation factor $\mathrm{A} \cdot \mathrm{H}^{1 / 2}$, where $\mathrm{A}$ is the door area and $\mathrm{H}$ its height. This parameter ranged approximately from $10^{-3}$ to $3.10^{2} \mathrm{~m}^{5 / 2}$. In the stable oscillatory combustion region, the authors found that the burning rate increased significantly with the ventilation parameter, well beyond the burning rate in open atmosphere. This result was associated with the dynamic balance between the rate of air supply and fuel supply. Moreover, the burning rate also increased with the compartment size in such a way that the maximum burning rate was up to 7.2 times the open burning rate for a compartment of $0.5 \mathrm{~m}$ in side. This effect indicated a strong influence of the compartment size on the fire behaviour. Later, Fleischman et al. [8] recorded similar results for another liquid fuel (heptane) located in a compartment of $1 \mathrm{~m} 3$ in volume equipped with small openings. For these experiments, the ventilation factor was in the range from 0.0039 to $0.071 \mathrm{~m}^{5 / 2}$. As with Takeda et al. [7], Fleischman et al. observed that the increase of mass loss rate was about 7 times greater than the same pool fire in open atmosphere. From both these studies, the main conclusion was that the compartment size could have a significant effect on the mass loss rate for small pool fire due to thermal radiation feedback.

More recently, Utiskul et al.[5] investigated the fire behaviour of heptane pool fires (6.5 to $19 \mathrm{~cm}$ in diameter) in a small-scale cubic compartment ( $40 \mathrm{~cm}$ in side) with top and bottom wall vents (total area varying from 2 to $240 \mathrm{~cm}^{2}$ ). In spite of a natural ventilation slightly different from that of the previous study (two openings versus only one), the authors observed very similar fire behaviours (steady burning, extinction...). A simplified theory based on a uniform-property compartment model was proposed to estimate the burning rates as a function of the oxygen concentration, the external heat flux and the free burning rate. Thanks to this approach, Utiskul et al. [2] simulated the burning rate of their fire experiments under limited ventilation.

In a well-controlled environment, many small-scale experiments ([9], [10], [11]) were carried out in a Fire Propagation Apparatus (ASTM E 2058) for various types of fuels (plastics, wood, liquid) including PMMA, plywood and heptane. In this enclosure, the oxygen concentration of the air surrounding the fuel sample was well controlled by means of a mixture of air/ $\mathrm{O}_{2} / \mathrm{N}_{2}$. This apparatus is particularly well designed 
to investigate fire behaviour under oxygen depletion. Under these conditions, a linear decrease of the fuel mass loss rate with the oxygen concentration was reported.

In a medium-scale room (about $34 \mathrm{~m}^{3}$ in volume) and using both natural and mechanical ventilation, Peatross et al.[3] investigated the effect of ventilation on the compartment fire environment. Diesel, wood cribs and polyurethane slabs were used as combustibles. In naturally ventilated fire tests, two layers were observed, both from the oxygen concentration and the temperature profiles. The oxygen concentration profiles indicated single-layer environments in all experiments using mechanical ventilation, so that the fire room could be considered similar to a well-stirred reactor. However, the thermal stratification suggested either one- or two-layers depending on the ventilation scenario. Moreover, experimental results showed that the burning rates had no dependence on the external radiation from the compartment but only on the fuel type and on the ventilation rate. The authors fit the experimental data with a linear correlation between the normalized burning rates per unit area and the oxygen concentration close to the flame base. This relationship between oxygen concentration and the mass loss rate was obviously consistent with the experimental results of Tewarson ([9], [10]) and Santo [11].

From the previous results, it can be noted that the influence of the oxygen concentration on the mass loss rate (and consequently on the heat release rate) can imply a significant increase in the fire duration in so far that flame extinction does not occur. Recently, full-scale experiments of mechanically-ventilated compartment fires were also conducted by IRSN [12] in a $120 \mathrm{~m}^{3}$ room using a dodecane fuel. Some of these experiments showed steady combustion regimes with limited pyrolysis, yielding fire durations up to 2.5 times the fire duration in free atmosphere, for the same amount of fuel and pool surface.

\section{EXPERIMENTS}

For many years, IRSN has been investigating the influence of limited ventilation on compartment pool fires equipped with mechanical ventilation systems in the framework of improving fire safety in nuclear plants. Two facilities have been used, both of them connected to a ventilation network. The first one (Fig. 1) involved a $400 \mathrm{~m}^{3}(7.5 \mathrm{~m}$ high) concrete fire room for which the airflow resistance is higher in the inlet branch that in the outlet branch. The second one (Fig. 2) involved a $120 \mathrm{~m}^{3}(4 \mathrm{~m}$ high) concrete fire room for which the inlet and outlet airflow resistances are of the same order of magnitude. Various experimental conditions were studied such as ventilation level, pool size and fuel type. Among these experiments, some provide very useful features to study the influence of oxygen depletion on the combustion, in particular quite a long time of quasi-steady combustion. Their main features are summarized in Table 1.

Table 1. Experimental conditions

\begin{tabular}{|c|c|c|c|c|c|c|c|}
\hline $\begin{array}{c}\text { Test } \\
\text { number }\end{array}$ & Reference & $\begin{array}{c}\text { Pool } \\
\text { surface } \\
\left(\mathrm{m}^{2}\right)\end{array}$ & Fuel & $\begin{array}{c}\text { Room } \\
\text { volume } \\
\left(\mathrm{m}^{3}\right)\end{array}$ & $\begin{array}{c}\text { Initial inlet air } \\
\text { mass flow-rate } \\
(\mathrm{kg} / \mathrm{s})\end{array}$ & $\begin{array}{c}\text { Initial fuel } \\
\text { mass (kg) }\end{array}$ & $\begin{array}{c}\text { Position of } \\
\text { the inlet } \\
\text { ventilation } \\
\text { branch }\end{array}$ \\
\hline 1 & {$[12]$} & 0.4 & HTP & 120 & 0.183 & 15.0 & high \\
\hline 2 & {$[12]$} & 0.4 & HTP & 120 & 0.337 & 15.7 & high \\
\hline 3 & {$[12]$} & 0.4 & HTP & 120 & 0.192 & 15.7 & high \\
\hline 4 & {$[12]$} & 0.2 & HTP & 120 & 0.187 & 7.2 & high \\
\hline 5 & {$[12]$} & 0.4 & HTP & 120 & 0.187 & 16.0 & low \\
\hline 6 & {$[12]$} & 0.4 & HTP & 120 & 0.067 & 15.8 & low \\
\hline 7 & {$[13][14]$} & 1 & HTP/TBP & 400 & 0.247 & 41.2 & high \\
\hline 8 & {$[13][14]$} & 0.4 & HTP/TBP & 400 & 0.4 & 16.7 & low \\
\hline 9 & {$[12]$} & 0.56 & oil & 120 & 0.12 & 20.0 & high \\
\hline
\end{tabular}


Most of the experiments used hydrogenated tetra-propylene (HTP) as a fuel but two of them involved a mixture of $70 \%$ vol HTP and 30\%vol tri-butyl-phosphate (TBP); oil was also used (MOBIL OIL DTE MEDIUM). Tests 5 and 6 use the same experimental protocol as tests 1-4 except for the position of the inlet ventilation duct, situated $0.7 \mathrm{~m}$ above the floor against $3.3 \mathrm{~m}$ for the "high" inlet position. The pool is located at the centre of the room, except for test 7 where the pool was put near a wall. Test 9 used the same experimental protocol as tests $1-4$ but the fuel was preheated up to $250^{\circ} \mathrm{C}$ before the test.

All the experiments were instrumented with several K-type thermocouples located along vertical trees; the estimated uncertainty on the mean gas temperature in the room is about $10 \mathrm{~K}$. The oxygen concentration (SERVOMEX 4100) was measured at three positions in the fire room: one was situated near the pool at $0.35 \mathrm{~m}$ above the floor; the two others located respectively $0.8 \mathrm{~m}$ above the floor and $0.7 \mathrm{~m}$ below the ceiling. From these two oxygen concentration measurements, the mean concentration in the fire room is estimated with an uncertainty around $1 \%$. Table 2 summarizes the main experimental results.

Table 2. Experimental results

\begin{tabular}{|c|c|c|c|c|c|}
\hline Test number & $\begin{array}{c}\text { Well-ventilated } \\
\text { mass loss rate } \\
\left(\mathrm{g} / \mathrm{m}^{2} / \mathrm{s}\right)\end{array}$ & $\begin{array}{c}\text { Steady-state } \\
\text { mass loss rate } \\
\left(\mathrm{g} / \mathrm{m}^{2} / \mathrm{s}\right)\end{array}$ & $\begin{array}{c}\text { Mean } \mathrm{O}_{2} \\
\text { during steady- } \\
\text { state } \\
(\% \mathrm{vol})\end{array}$ & $\begin{array}{c}\mathrm{O}_{2} \text { near flame } \\
\text { base during } \\
\text { steady-state } \\
(\% \mathrm{vol})\end{array}$ & $\begin{array}{c}\text { Mean gas } \\
\text { temperature } \\
\text { during steady- } \\
\text { state } \\
(\mathrm{K})\end{array}$ \\
\hline 1 & 34.7 & 10.0 & 14.9 & 14.2 & 435 \\
\hline 2 & 34.7 & 15.0 & 17.2 & 15.5 & 470 \\
\hline 3 & 34.7 & 10.5 & 14 & 14.2 & 435 \\
\hline 4 & 28.7 & 14.5 & 18.0 & 16.3 & 400 \\
\hline 5 & 34.7 & 16.0 & 11.7 & 16.1 & 475 \\
\hline 6 & 34.7 & 10.5 & 13.2 & 13.5 & 435 \\
\hline 7 & 38.2 & 8.3 & 14.9 & $\mathrm{n} / \mathrm{a}$ & 448 \\
\hline 8 & 31.8 & 20.0 & 16.6 & 18.5 & 399 \\
\hline 9 & $28.6^{(1)}$ & 9.3 & 14.3 & 14.8 & 428 \\
\hline
\end{tabular}

(1) Estimated value from transformer oil [4].

All the tests exhibited limited excess gas temperature; the maximum mean gas temperature ( $475 \mathrm{~K})$ was reported in test 5 and is well below usual values found during flashover. Experiments 6 and 7 yielded extinguishment due to lack of oxygen, whereas the other experiments yielded sustained combustion until fuel exhaustion.
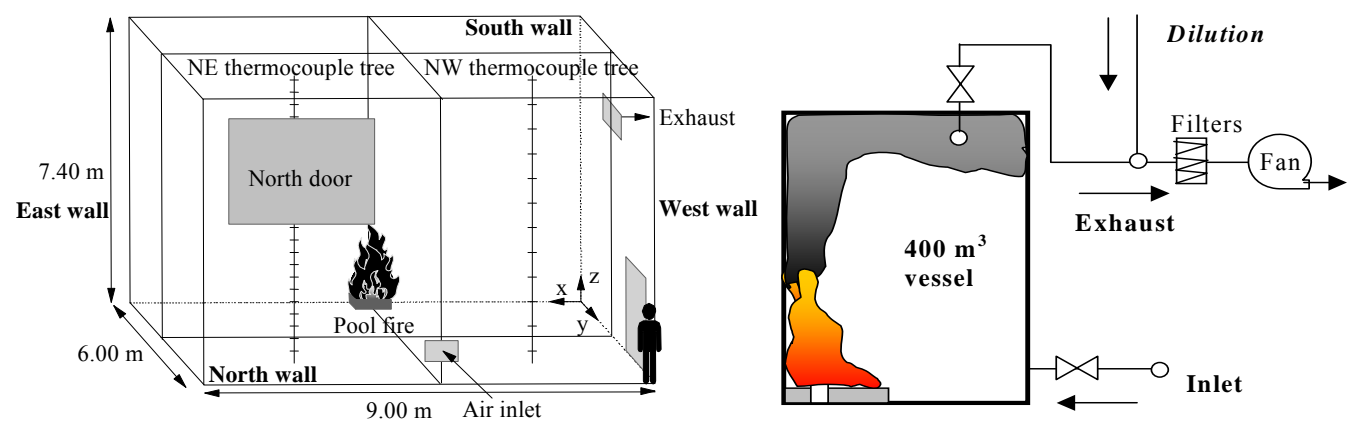

Fig. 1. Facility of $400 \mathrm{~m}^{3}$ (test numbers $7 \& 8$ ). 


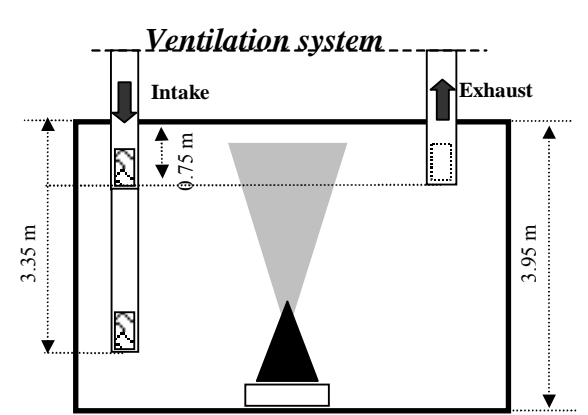

Side view of fire room

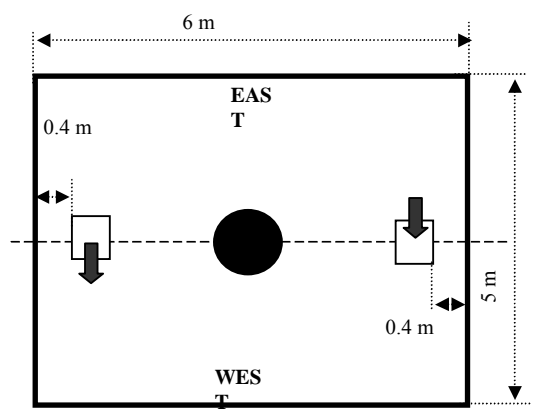

Top view of fire room

Fig. 2. Facility of $120 \mathrm{~m}^{3}$ (test numbers 1 to $6 \& 9$ ).

\section{WELL-STIRRED REACTOR APPROACH}

The well-stirred reactor approach has been used to study the overall behaviour of room fires [3][5]. Following Utiskul et al.[5], it is applied here to the mechanically-ventilated case to provide insight on the quasi steady-state pyrolysis rate.

\section{Quasi steady-state of mechanically ventilated fires}

For the simplest ventilation networks, the pressure can be related to the inlet and outlet flows by a Bernoulli equation. Both pressure drops due to the airflow resistance and overhead due to fans are then supposed to follow a quadratic relationship with the air flow rate q. The ventilation network is therefore represented by six variables: the upstream (overhead) pressure $P_{\text {up }}$, the inlet airflow resistance $R_{u p}$, the inlet air mass flowrate $q_{\text {in }}$, the downstream pressure $P_{\text {down }}$, the outlet airflow resistance $R_{\text {down }}$ and the outlet air mass flow-rate $\mathrm{q}_{\text {out }}$. The pressure in the compartment is related to these variables by

$$
P_{\text {up }}-P=R_{\text {up }} q_{\text {in }}^{2} / \rho_{\infty}, \quad P-P_{\text {down }}=R_{\text {down }} q_{\text {out }}^{2} / \rho
$$

The airflow resistances of the network can be eliminated from Eq. 1 when introducing the initial conditions in the fire room, that is $\mathrm{P}=\mathrm{P}_{0}, \mathrm{q}_{\mathrm{in}}=\mathrm{q}_{\text {out }}=\mathrm{q}_{0}$ and $\rho=\rho_{\infty}$. Further assuming $\rho T=\rho_{\infty} T_{\infty}$, Eq. 1 rewrites as

$$
\frac{P_{\text {up }}-P}{P_{\text {up }}-P_{0}}=q_{\text {in }}^{2} / q_{0}^{2}, \quad \frac{P-P_{\text {down }}}{P_{0}-P_{\text {down }}}=\frac{T}{T_{\infty}} q_{\text {out }}^{2} / q_{0}^{2}
$$

During the quasi steady-state, the temperature in the compartment is supposed to vary slowly, so that $\mathrm{T}$ is taken as an input parameter. As the fuel mass flow-rate is small compared to the ventilation flows, the mass balance during the quasi steady-state imposes $q_{\text {in }}=q_{\text {out }}=\bar{q}$. Eliminating the room pressure in Eq. 2 therefore provides the quasi steady-state ventilation mass flow-rate.

$\bar{q}=q_{0}\left[1+\left(\frac{T}{T_{\infty}}-1\right) \frac{P_{0}-P_{\text {down }}}{P_{\text {up }}-P_{\text {down }}}\right]^{-1 / 2}$

The steady-state ventilation mass flow-rate only deviates from the initial flow-rate by a function that weakly depends on the temperature and on the initial pressures in the ventilation network. Therefore, the pressure ratio in Eq. 3 represents the dissymmetry of the network airflow resistances. 
The fire is modelled assuming a constant combustion heat. The literature reports a linear dependency of the mass loss rate on the oxygen content [10][11][3][5]. Without loss of generality, this dependency is written as

$\dot{m}^{\prime \prime}=\dot{m}_{\infty}^{\prime \prime}\left[(1+\alpha) \frac{Y}{Y_{\infty}}-\alpha\right]$

where the coefficient $\alpha$ is representative of the rate of decrease of pyrolysis rate with the oxygen concentration. The original correlation of Peatross and Beyler [3] corresponds to $\alpha=1.10$, but some studies (e.g.[5] [16]) also use $\alpha=0$. Equation 4 represents the effect of vitiation on the heat release rate. One possible mechanism of the limited pyrolysis rate in a vitiated environment has been proposed [11]: as the oxygen content in the vicinity of the fire decreases, the flame becomes less sooty and consequently less radiative feed-back is experienced by the pool, therefore decreasing the pyrolysis rate.

The oxygen sink term is directly linked to the fuel pyrolysis through the oxygen-to-fuel mass ratio $s=\Delta H_{c} / \Delta H_{O 2}$, so that the oxygen mass balance becomes

$\rho V \frac{d Y}{d t}=-A_{f} \operatorname{sim}_{\infty}^{\prime \prime}\left[(1+\alpha) Y / Y_{\infty}-\alpha\right]+q_{\text {in }} Y_{\infty}-q_{\text {out }} Y$

Dividing Eq. 5 by $Y_{\infty} q_{0}$ provides the non-dimensional form of oxygen conservation. The Global Equivalence Ratio (GER) $\Phi=A_{f} m_{\infty}^{\prime \prime} s / q_{0} Y_{\infty}$ is introduced. It should be noted that this definition of the global equivalence ratio only uses initial values and fuel properties. Equation (5) is rewritten as

$\frac{\rho V}{q_{0}} \frac{d\left(Y / Y_{\infty}\right)}{d t}=-\Phi\left[(1+\alpha) \frac{Y}{Y_{\infty}}-\alpha\right]+\frac{q_{\text {in }}}{q_{0}}-\frac{q_{\text {out }}}{q_{0}} \frac{Y}{Y_{\infty}}$

During the quasi steady-state, $q_{\text {in }}=q_{\text {out }}=\bar{q}$ and the mean oxygen content $\bar{Y}$ is therefore given by

$$
\frac{\bar{Y}}{Y_{\infty}}=\frac{\alpha \Phi+\bar{q} / q_{0}}{(1+\alpha) \Phi+\bar{q} / q_{0}}
$$

Combining equations (4) and (7) provides $\overline{\dot{m}}^{\prime}$, the fuel mass loss rate during the quasi steady-state:

$$
\overline{\dot{m}}^{\prime \prime}=\dot{m}_{\infty} \frac{1}{1+(1+\alpha) \Phi \frac{q_{0}}{\bar{q}}}
$$

Equation 8 provides a simple way to characterize the influence of experimental conditions on the mass loss rate. From Eq. 8, the experimental conditions are mostly embedded in a unique parameter, the global equivalence ratio, whereas the parameters related to the ventilation network features and to the temperature play a second-order role. As long as the room temperatures do not cause significant additional heat flux to the fuel surface and therefore additional pyrolysis, Eq. 8 provides a dimensional relationship for the fuel mass loss rate. 


\section{Experimental comparison}

Figure 3 illustrates the decisive influence of the global equivalence ratio on the steady-state mass loss rate. The experimental results are presented as $\dot{m}_{\infty}^{\prime \prime} / \overline{\dot{m}}^{\prime \prime}=f(\Phi)$ in order to investigate the linearity and the slope of the denominator of Eq. 8. The experimental points are constructed using the experimental mass loss rate values averaged during the quasi steady-state period ; the well-ventilated mass loss rates are known from hood experiments. The global equivalence ratio is calculated from the experimental initial conditions; $\Delta \mathrm{H}_{\mathrm{O} 2}=13.1 \mathrm{MJ} / \mathrm{kg}$ was used for all the combustibles and the heat of combustion is known from cone calorimeter measurements: $\Delta \mathrm{H}_{\mathrm{c}}(\mathrm{TPH})=39 \mathrm{MJ} / \mathrm{kg}, \Delta \mathrm{H}_{\mathrm{c}}(\mathrm{TBP} / \mathrm{TPH})=35 \mathrm{MJ} / \mathrm{kg}$ and $\Delta \mathrm{H}_{\mathrm{c}}($ oil $)=$ $39 \mathrm{MJ} / \mathrm{kg}$. Table 3 provides the calculated values. The theoretical line is calculated with a dissymmetry factor of 0.5 and considering a room temperature of $430 \mathrm{~K}$. Both these hypotheses represent roughly the experimental conditions (see Table 2). They imply setting $\bar{q} / q_{0}=0.9$ in Eq. 8. The coefficient $\alpha=1.1$ is used according to [3].

Table 3. Global equivalence ratio

\begin{tabular}{|c|c|c|c|c|c|c|c|c|c|}
\hline $\begin{array}{c}\text { Test } \\
\text { number }\end{array}$ & 1 & 2 & 3 & 4 & 5 & 6 & 7 & 8 & 9 \\
\hline$\Phi$ & 0,968 & 0,527 & 0,926 & 0,392 & 0,951 & 2,66 & 1,73 & 0,365 & 0,91 \\
\hline
\end{tabular}

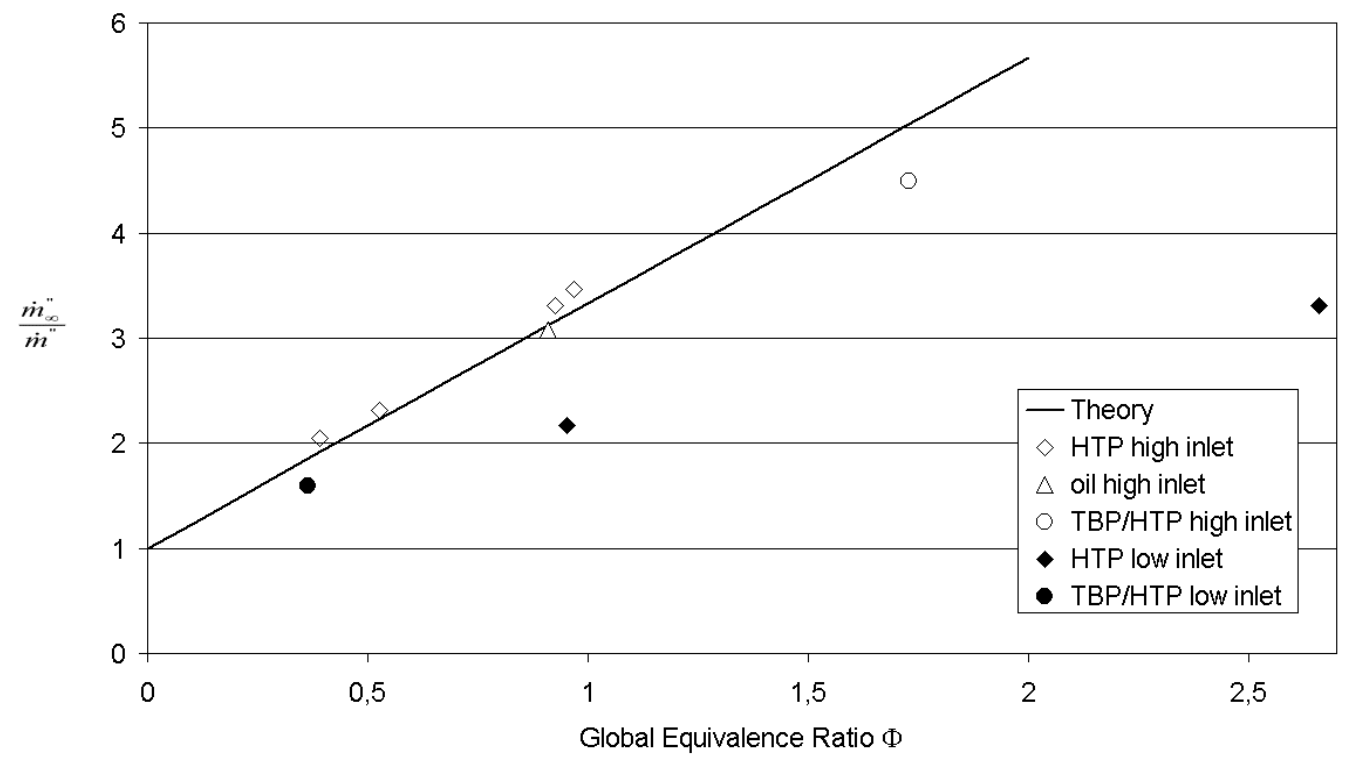

Fig. 3. Evolution of the steady-state fuel mass loss rate with the global equivalence ratio.

Figure 3 suggests two different behaviours: experiments with elevated air inlet (open symbols) fit remarkably well to the theoretical line whereas the experiments with inlet near the floor (full symbols) show significant deviation from it. Nevertheless, the test 6 with the highest GER presented a very short steady-state (about 60s) due to early extinction. The uncertainty for this experimental value is therefore quite high and more experimental confirmation ought to be gathered before a definitive conclusion can be drawn. Figure 4 presents the experimental mass loss rates as a function of the steady-state oxygen concentration measured near the flame base. The results are close to the correlation of Peatross and Beyler [3]. It is remarkable that even the experiments with low inlet position fit this correlation properly, even though they deviated from the theory in Fig. 3. This questions the assumption of a well-stirred reactor for such a configuration. Indeed, the behaviour of room fires with low inlet could be close to that of naturally-ventilated fires, where the available oxygen near the flame base is significantly higher than the mean oxygen concentration [3]. The differences between the oxygen content measured near the flame base 
and the mean oxygen content provided in Table 2 supports this assumption (test 6 experienced early extinction and seems to be an exception).

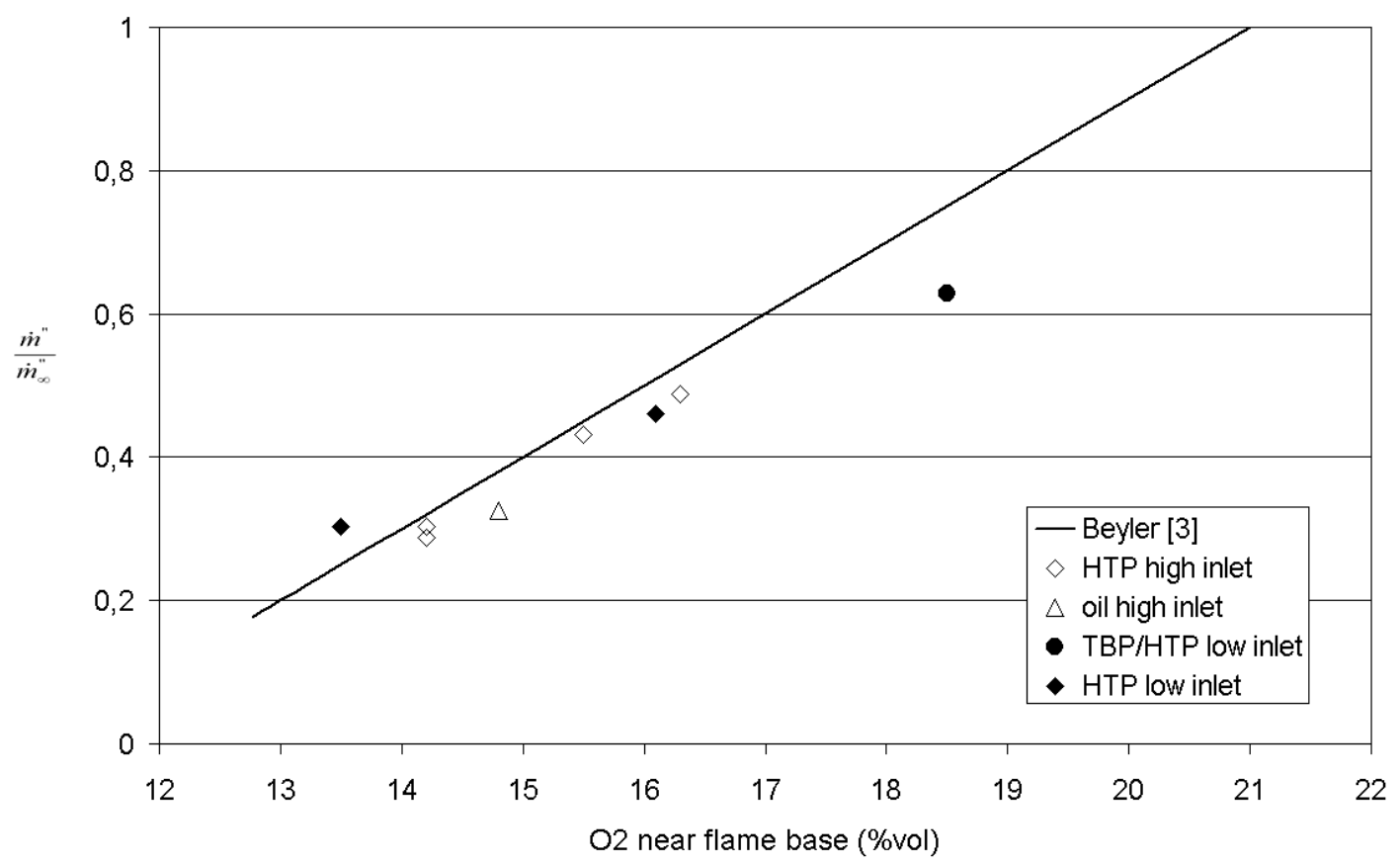

Fig. 4. Evolution of the steady-state fuel mass loss rate with oxygen.

\section{SYLVIA CODE: ZONE MODELLING}

Clearly from the previous well-stirred reactor analysis, a proper representation of the oxygen supplying the fire is a key condition to estimate the rate of heat release. Consequently, a zone-model approach is adopted to account for possible oxygen stratification. A better estimation of the oxygen near the flame is expected. The Peatross and Beyler correlation is embedded in the modelling and the comparison with the experiments gives further insight to the dynamic behaviour of the mass loss rate and of heat release rate in low-oxygen conditions.

\section{Model overview}

The SYLVIA code has been recently developed at IRSN. It combines a classical zone model [6] and a detailed representation of the ventilation network. Basically, the ventilation network is described on the one hand with branches where the mass flow-rates are the main unknowns and on the other hand with nodes where the scalar variables - gas pressure and temperature, species mass fractions- are assumed to be uniform within control volumes. The branches are associated to a generalized version of the Bernoulli equation accounting for momentum inertia. The conservation equation of mass, species and energy are solved for each node following the classical zones-model approach. The walls are meshed in two dimensions but the heat conduction equation is solved only along the wall depth. A first-order, finitevolume, graded meshing approach is used to account for steep temperature gradients in the walls. The heat transfer modelling include natural convection and forced convection heat transfer, with coefficients correlated in the literature. The model for the thermal radiation exchanges is basically a grey-gas pointsource model: the fire is represented as a punctual source emitting a radiation power that is a prescribed fraction of the heat release rate. Absorption and emission by the gases account for the concentration in water vapour, carbon dioxide and soot.

In SYLVIA, the fuel pyrolysis is calculated to account for vitiation. Two main features are incorporated in the model. At ignition, an increasing function of time $\mathrm{g}(\mathrm{t})$ accounts for the heat-up of the pool, in accordance to hood experiments. The influence of oxygen depletion is given by the Peatross and Beyler correlation [3], so that the pool mass loss rate is calculated as: 
$\dot{m}^{\prime \prime}=g(t) \cdot \dot{m}_{\infty}^{\prime \prime}\left[(1+\alpha) \frac{Y}{Y_{\infty}}-\alpha\right]$

with $\alpha=1.1$ and $\mathrm{g}(\mathrm{t})$ reaching $90 \%$ within 300 s. The oxygen content $\mathrm{Y}$ used in Eq. 9 is given by interpolation between the lower zone and upper zone composition:

$$
Y= \begin{cases}Y_{\text {inf }} & \text { if } z_{\text {int }}<z_{\text {pool }} \\ Y_{\text {sup }} & \text { if } z_{\text {int }}>z_{\text {pool }}+h_{f}(10) \\ Y_{\text {inf }}\left(z_{\text {int }}-z_{\text {pool }}\right) / h_{f}+Y_{\text {sup }}\left(z_{\text {pool }}+h_{f}-z_{\text {int }}\right) / h_{f} & \text { otherwise }\end{cases}
$$

The flame height $h_{f}$ is calculated from the correlation of Heskestad [15]. The heat of combustion is constant during the combustion and is deduced from hood experiments, performed under well-ventilated conditions. As the temperatures in the experiments considered here are relatively low (under $200^{\circ} \mathrm{C}$ ), the influence of an external flux on the pyrolysis rate is not accounted for in the pyrolysis model. Fire extinction occurs either due to lack of fuel or based on a critical surface pyrolysis rate criterion. In this last case, a unique threshold value of $10 \mathrm{~g} / \mathrm{m}^{2} / \mathrm{s}$ was used for experiments using HTP and TBP/HTP; no extinction data are available for the oil experiment (test 9), therefore this feature is not modelled for this test.

Eventually, the whole set of equations constitute an ordinary differential equation system that is solved implicitly using a BDF technique suited to stiff problems and coupled to an iterative Newton-Raphson resolution.

\section{Experimental comparison}

The model has been compared to the experiments. Figures 4 to 9 illustrate the dynamic behaviour of pool fires in a mechanically-ventilated compartment. For the tests with the inlet near the ceiling (exemplified by Fig. 5. to Fig. 10 for the tests 1 and 2), the calculated oxygen and carbon dioxide fit the experimental data with good agreement. As the code uses a constant chemical reaction estimated from well-ventilated conditions, this good agreement suggests that there is no noticeable influence of the confinement on the combustion. Furthermore, as the heat of combustion is directly linked to the oxygen consumption and to the emission of carbon dioxide, this also suggests that the rate of heat released is properly calculated. On the contrary, calculations for the tests with a low inlet can yield significant discrepancies even though a zone approach was adopted. This discrepancy is displayed in Fig. 9 and Fig. 10 for test 5. The calculated burning rate is under-estimated whereas the mean oxygen content is over-estimated. This does not contradict the Peatross and Beyler correlation as the oxygen content near the pool is lower in the calculation than in the experiments. Indeed, the calculation estimates a local oxygen concentration near the pool of $15.2 \%$ against the experimental $16.1 \%$. Except for the inlet position, the tests 1 and 5 are similar. But, the maximum mean $\mathrm{CO} / \mathrm{CO}_{2}$ content in the compartment was 10 times higher in test 5 than in test 1 (respectively $2 \%$ and $0.2 \%$ ). This indicates a less efficient reaction of combustion that could explain the discrepancy between the numerical results and the experimental data in test 5 . As the chemistry is imposed in the calculation with an efficiency of combustion equal to the well-ventilated case, the consumption of oxygen is over-estimated. 


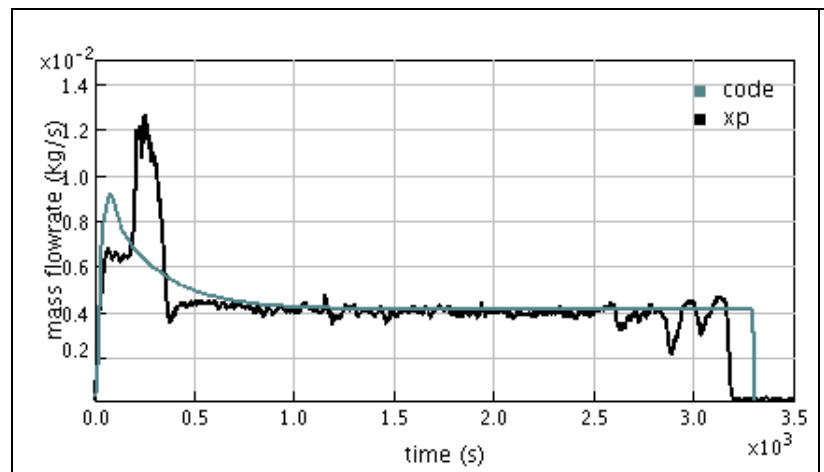

Fig. 5. Fuel pyrolysis rate during test 1

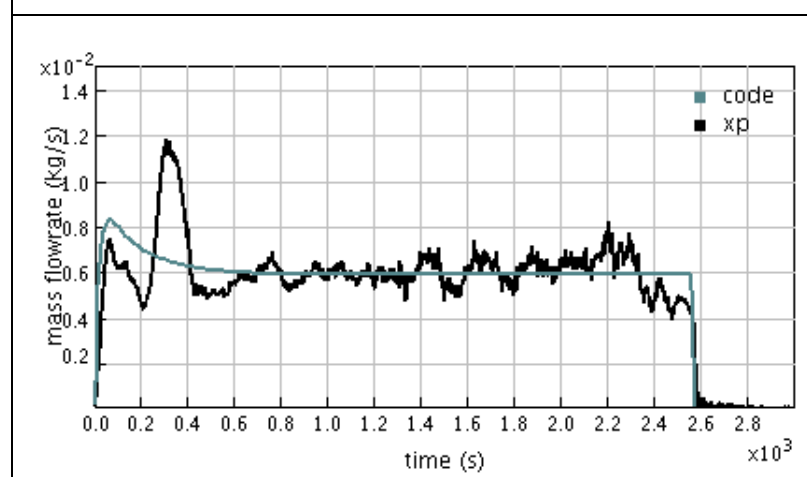

Fig. 7. Fuel pyrolysis rate during test 2

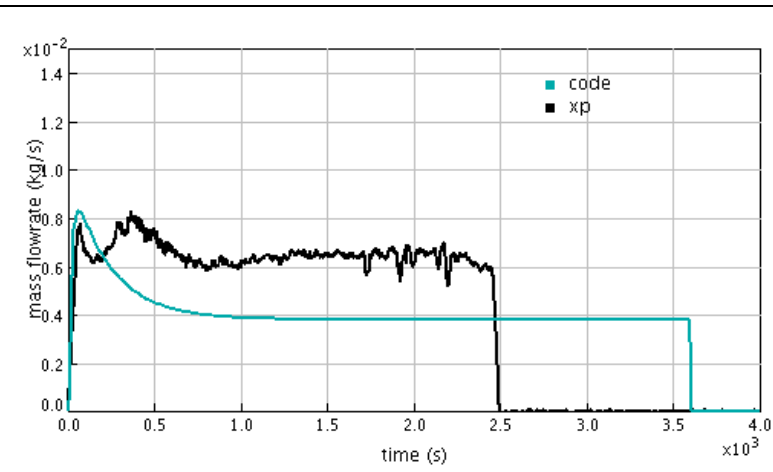

Fig. 9. Fuel pyrolysis during test 5

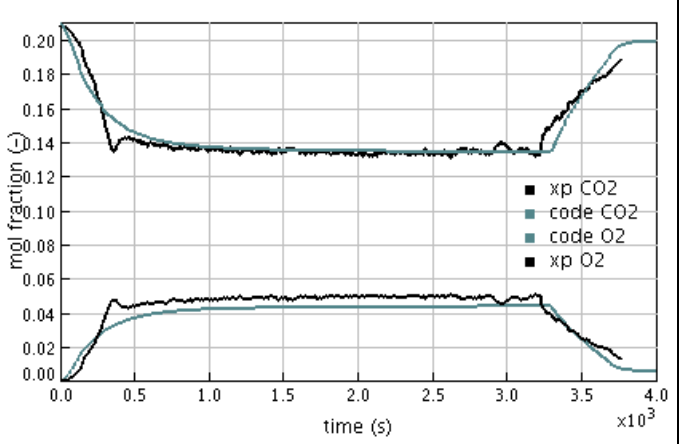

Fig. 6. Oxygen and $\mathrm{CO}_{2}$ content during test 1

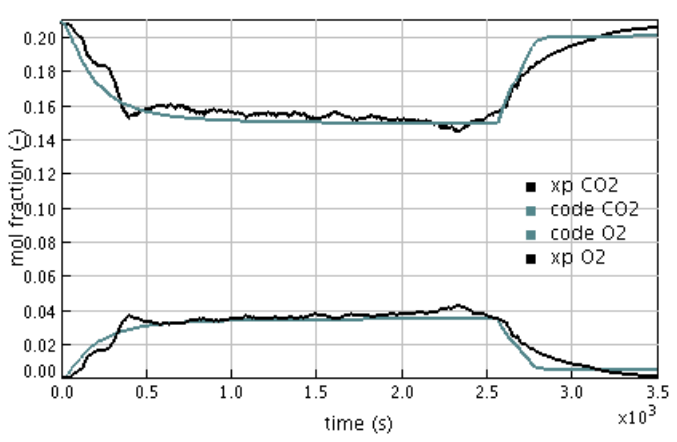

Fig. 8. Oxygen and $\mathrm{CO}_{2}$ content during test 2

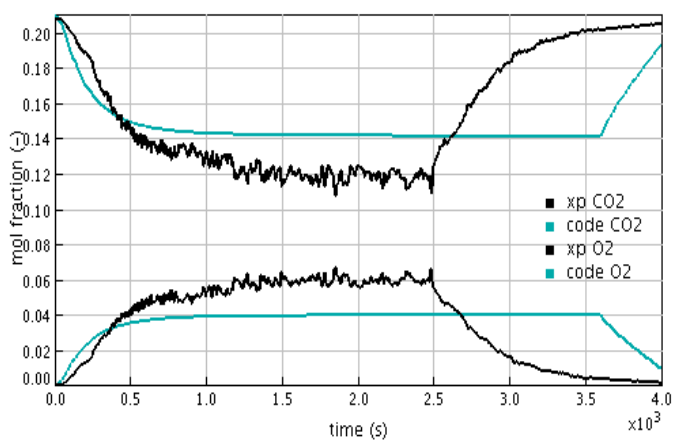

Fig. 10. Oxygen and $\mathrm{CO}_{2}$ content during test 5

The effect of vitiation on the fire duration can play two different roles, depending on the global equivalence ratio. For limited values of the global equivalence ratio, the decrease of the pyrolysis rate induces a longer fire duration than in the open atmosphere. On the contrary, for a given value of the global equivalence ratio, the pyrolysis rate is so low that no combustion can be sustained and extinction occurs. The tests 6 and 7 typically experienced this early extinction phenomenon. As shown on Fig. 11, both these phenomena are properly predicted using a simple zone approach coupled to the Peatross and Beyler correlation. However, the model largely over-predicts the fire duration of test 5. 


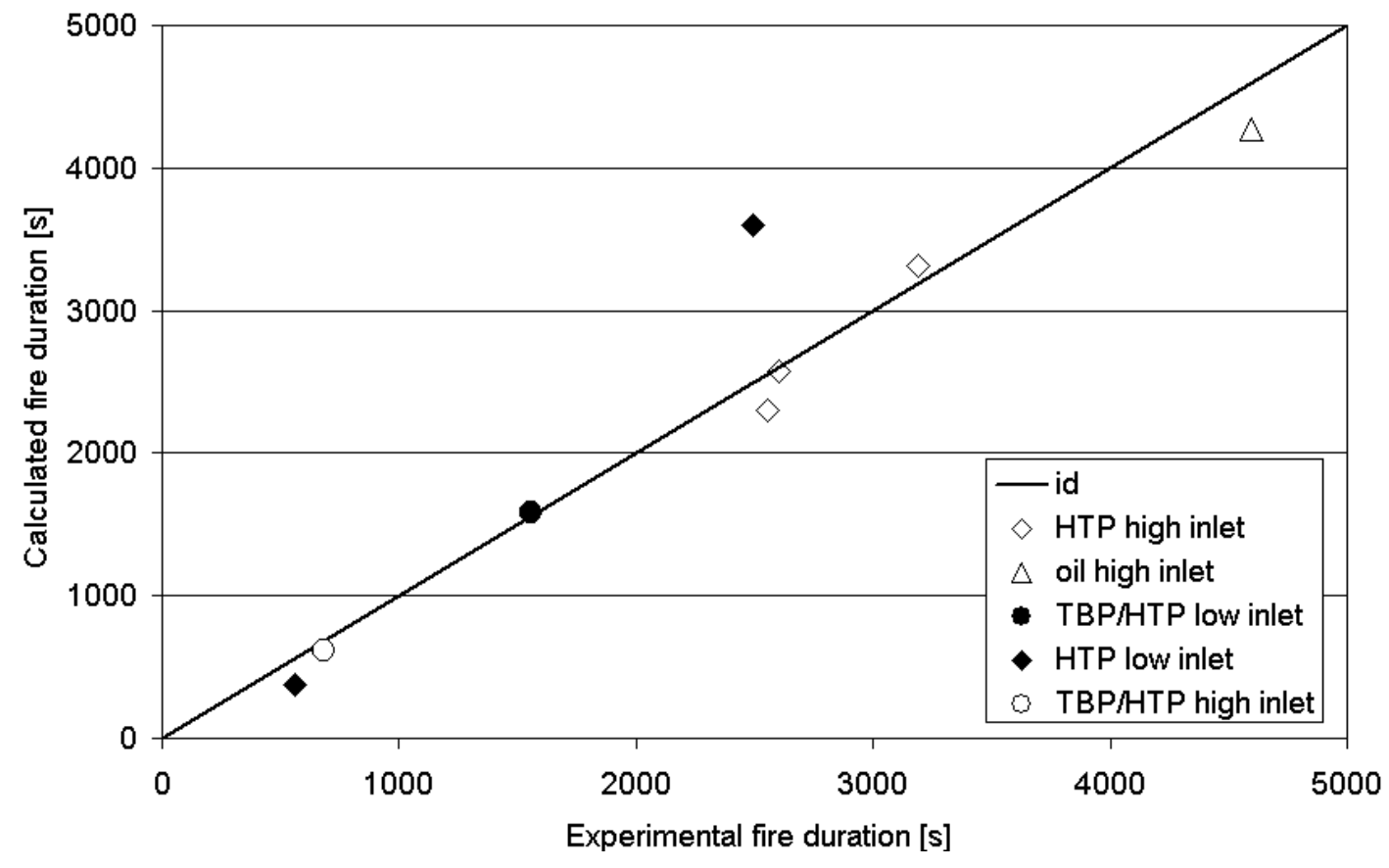

Fig. 11. Experimental versus calculated fire durations.

\section{CONCLUDING REMARKS}

From the nine pool-fire experiments presented, the correlation of Peatross and Beyler appears to apply with good confidence to mechanically-ventilated fires as long as the oxygen concentration considered in the correlation is representative of the concentration near the flame base. The problem for modelling the burning rate in a vitiated environment arises from the determination of this local oxygen content. Experimentally, when the ventilation inlet is high, the compartment behaves as a well-stirred reactor and the burning rate can be readily estimated from Eq. 8. As no significant decrease in the heat of combustion has been observed, the estimation of the rate of heat release is straightforward in this case. When the inlet position is low, the oxygen concentrations in the compartment tend to be stratified much like naturallyventilated fires. In this case, the mean oxygen concentration can be significantly lower than the oxygen near the flame and the well-stirred reactor approach breaks down. A zone modelling approach provides a better estimation of the oxygen content surrounding the pool for both positions of the inlet. Still, it seems that the oxygen gradient in the low-inlet case also influences the combustion efficiency, a feature that is not accounted for in our zone model.

Comparing the tests 1 and 5, it is remarkable that the low-inlet case both provides a higher mass loss rate and a reduced combustion efficiency than the high-inlet case. The decrease of the burning rate with the oxygen concentration is generally attributed to a less intensive combustion due to oxygen depletion, leading to reduced heat fluxes to the pool surface. One would therefore expect the burning rate and the combustion efficiency to vary in the same way. Presumably, this apparent paradox is again due to oxygen gradients within the compartment: the available oxygen near the pool is significantly higher than the mean oxygen content, leading to an increased mass loss rate compared to the high-inlet case. In essence, a significant part of the flame is located in a more vitiated environment, yielding less efficient combustion and producing a larger amount of carbon monoxide. However, as the upper part of the flame contributes weakly to the radiative heat flux to the pool surface, the mass loss rate is not influenced significantly by the decrease of the combustion efficiency.

The analysis presented here is only limited to cases where the gas temperatures are not very high in the room, so that the radiation feedback from the environment to the pool surface can be supposed to be negligible. This provides helpful insight into the oxygen-depletion effect on the burning rate but cannot be extended directly to any confined pool fire. In particular, the leading phenomena could be quite different in near-flashover or post-flashover conditions. The question also arises for the near-extinction regime. In this 
case, the flame emissivity is known to decrease, eventually causing extinction, so that the pool is more prone to the effect of external fluxes. The same conclusion can be drawn for small pool fires [5][16]. The Peatross and Beyler correlation has not been validated for this domain, in which the linear dependency of the mass-loss rate on the oxygen concentration could fail. Other limitations could arise from particular fuels (especially charring materials) or fuel configurations: on this last point, little knowledge has been acquired on the effect of vitiation on vertical fires.

\section{REFERENCES}

[1] Babrauskas, V., Peacock, R.D., (1992), Heat Release Rate: The Single Most Important Variable in Fire Hazard, Fire Safety Journal 18: 255-272, doi:10.1016/0379-7112(92)90019-9.

[2] Babrauskas, V., "Heat Release Rates", The SFPE Handbook of Fire Protection Engineering ( $3^{\text {rd }}$ ed), DiNenno P.J. (ed.), National Fire Protection Association, Quincy, MA 02269, 2002, p. 3/1, .

[3] Peatross, M. J., Beyler, C. L., "Ventilation Effects on Compartment Fire Characterization," Fire Safety Science -- Proceedings of the Fifth International Symposium, International Association for Fire Safety Science, 1997, pp. 403-414, doi:10.3801/IAFSS.FSS.5-403.

[4] Babrauskas, V., Heat Release Rate, DiNenno P.J. (ed.), 1992, pp. 3/1.

[5] Utiskul, Y., Quintiere, J. G., Rangwala, A. S., Ringwelski, B. A., Wakatsuki, K., Naruse, T., (2005), Compartment Fire Phenomena under Limited Ventilation, Fire Safety Journal 40: 367390, doi:10.1016/j.firesaf.2005.02.002.

[6] Quintiere, J. G., Fundamental of Fire Phenomena, John Wiley and Sons, Chichester, 2006.

[7] Takeda, H., Akita, K., "Critical Phenomenon in Compartment Fires with Liquid Fuels", Proceedings of the Eighteenth Symposium (International) on Combustion, Combustion Institute, 1981, pp. 519-527, doi:10.1016/S0082-0784(81)80057-4.

[8] Fleischmann, C. M., Parkes, A. R., "Effects of Ventilation on Compartment Enhanced Mass Loss Rate", Fire Safety Science -- Proceedings of the Fifth International Symposium, International Association for Fire Safety Science, 1997, pp. 415-426, doi:10.3801/IAFSS.FSS.5-415.

[9] Tewarson, A., Pion, R. F., (1976), Flammability of Plastics - I. Burning Intensity, Combustion and Flame 26: 85-103, doi:10.1016/0010-2180(76)90059-6.

[10] Tewarson, A., Lee, J. L., Pion, R. F., "The Influence of Oxygen Concentration on Fuel Parameters for Fire Modeling", Proceedings of the Eighteenth Symposium (International) on Combustion, Combustion Institute, 1981, pp. 563-570, doi:10.1016/S0082-0784(81)80061-6.

[11] Santo, G., Tamanini, F., "Influence of Oxygen Depletion on the Radiative Properties of PMMA", Proceedings of the Eighteenth Symposium (International) on Combustion, Combustion Institute, 1981, pp. 619-631, doi:10.1016/S0082-0784(81)80067-7.

[12] Pretrel, H., Querre, P., "Experimental Study of Burning Rate Behaviour in Confined and Ventilated Fire Compartments", Fire Safety Science -- Proceedings of the Eight International Symposium, International Association for Fire Safety Science, 2005, pp. 1217-1229, doi:10.3801/IAFSS.FSS.8-1217.

[13] Audouin, L., Tourniaire, B., "Incident heat fluxes from pool fires close to a wall in a well-confined compartment", $8^{\text {th }}$ int. conference on nuclear engineering, 2000.

[14] Pretrel, H., Such, J.M., Effect of ventilation procedures on the behaviour of a fire compartment scenario, Nuclear engineering and design 23:2155-2169, doi:10.1016/j.nucengdes.2005.03.003.

[15] Heskestad, G., "Fire plumes, flame heights and air entrainment", The SFPE Handbook of Fire Protection Engineering ( $3^{\text {rd }}$ ed), DiNenno P.J. (ed.), National Fire Protection Association, Quincy, MA 02269, 2002, p. 2/1

[16] Quintiere, J.G., Rangwala, A.S., (2004), A theory for flame extinction based on flame temperature, Fire and Materials 28:387-402, doi:10.1002/fam.835. 